\title{
Six months' treatment with lifitegrast in patients with moderate-to-severe symptomatic dry eye: a retrospective chart review
}

This article was published in the following Dove Press journal: Clinical Ophthalmology

\author{
Richard Teofilo Atallah' \\ Maria Virginia Castanos ${ }^{2,3}$ \\ Richard Najac ${ }^{2}$ \\ Eric Donnenfeld ${ }^{4}$ \\ 'Rutgers New Jersey Medical School, \\ Newark, NJ, USA; ${ }^{2}$ Department of \\ Research, Eye and Ear Infirmary of Mount \\ Sinai, New York, NY, USA; ${ }^{3}$ Department \\ of Ophthalmology, New York Eye \& Ear \\ Infirmary of Mount Sinai, New York, NY, \\ USA; ${ }^{4}$ Ophthalmic Consultants of Long \\ Island, New York University Medical \\ Center, Garden City, NY, USA
}

Purpose: To evaluate 6-month treatment benefits with lifitegrast ophthalmic solution 5\% in symptomatic dry eye patients.

Setting: Retrospective chart analysis.

Method: A retrospective chart review was conducted in 168 patients (111 females and 57 males) who presented with symptoms of chronic dry eye disease and were treated with lifitegrast $5 \%$ ophthalmic solution for 6 months. Collected symptom data included improvement of eye dryness, tearing, eye pain, fluctuation in vision, foreign body sensation, itching, grittiness, burning and contact lens intolerance if applicable. Collected clinical signs included changes in superficial punctate keratitis, corneal fluorescein staining, conjunctival hyperemia and presence of tear debris.

Results: Treatment with lifitegrast ophthalmic solution 5\% twice daily for 6 months significantly improved majority of dry eye symptoms reported by patients. Improvements were also observed in corneal and conjunctival staining and tear debris for most of the patients reviewed.

Conclusion: Treatment with lifitegrast twice a day for 6 months improved both signs and symptoms of chronic dry eye.

Keywords: lifitegrast, integrin antagonist, dry eye disease, DED, eye dryness, inflammation, ocular surface disease

\section{Introduction}

Dry eye disease (DED) is a complex, multifactorial ocular surface disease characterized by the loss of homeostasis of the tear film and accompanied by ocular symptoms. Ocular surface inflammation plays a very important role in the pathogenesis of DED. ${ }^{1}$ Although several dry eye management algorithms have been developed, it has been a challenge to adequately manage this highly prevalent and highly symptomatic disease due to the lack of treatment modalities that are efficacious in symptom relief. Lifitegrast ophthalmic solution 5\% (Xiidra ${ }^{\circledR}$, Shire plc) was approved by the US FDA in 2016 for the treatment of both signs and symptoms of DED. Lifitegrast is a novel small-molecule lymphocyte function-associated antigen-1 (LFA-1) antagonist that targets a key step in the dry eye inflammatory cycle by blocking the binding of intercellular adhesion molecule 1 (ICAM-1) to the integrin LFA-1 on the T-cell surface inhibiting T-cell-mediated inflammation. ${ }^{2,3}$ Although the lifitegrast clinical development program generated convincing efficacy and safety data of this novel therapy in 5 well-designed, 3-month efficacy and 1-year safety trials, ${ }^{4-6}$ there have
Correspondence: Eric Donnenfeld Ophthalmic Consultant of Long Island, 7II Stewart Ave \#I60, Garden City, NY II530, USA

Email ericdonnenfeld@gmail.com 
been no available clinical data on the effects of treatment with lifitegrast beyond 3 months. Therefore, the question remains whether the prolonged treatment with lifitegrast will provide additional benefits. DED is a chronic and perhaps progressive condition especially among more symptomatic patients ${ }^{7}$ and very likely requires continued treatment. In this retrospective chart review, we evaluated the effect of 6-month treatment with lifitegrast in patients with moderateto-severe symptomatic DED.

\section{Patients and methods}

\section{Patients}

The medical records of patients diagnosed with DED and treated with lifitegrast for 6 months or longer between September 2016 and February 2018 were reviewed. Posttreatment visits were scheduled at 1,2, 3 and 6 months. Inclusion criteria included patients aged 30 years and older who were diagnosed with moderate-to-severe DED by clinical examination and by patient-reported dry eye symptom defined by $>4$ on a visual analog scale of 1-10. Exclusion criteria included patients with mild DED or with recent history ( $<3$ months) of ocular surgery.

\section{Design and treatment}

This is a retrospective chart analysis to evaluate the efficacy and safety of lifitegrast ophthalmic solution 5\% (Xiidra, Shire plc) self-administered twice daily for 6 months for the treatment of moderate and severe DED. This study is exempted by 45 CFR 46.101(b). No patient consents were required. All data were recorded in such a manner that subjects could not be identified directly or through identifiers linked to their records. The study was conducted at two clinical practices in the states of New York and New Jersey.

Retrospective data collected included patient demographics, medical history, previous and current ocular treatment, clinical diagnosis and presentations, comorbidity, clinical signs and patient-reported symptoms that were consistent with DED. Patient-reported treatment side effects that may or may not be associated with lifitegrast treatment were also collected. Clinical signs evaluated and included in the review were superficial punctate keratitis (SPK), conjunctival staining (lissamine green), conjunctival hyperemia, tear meniscus, presence of debris in tears and best-corrected visual acuity (BCVA). Only treatment results at the 6-month visit were reported. Treatment effects were categorized into no improvement, minor, moderate and significant improvement based upon the same VAS used prior to the lifitegrast therapy of $1-10$ with 0 as no improvement; $1-3$ as minor improvement; 4-6 as moderate improvement and $7-10$ as significant improvement.

\section{Results}

A total of 168 patients were included in the chart review (111 [66\%] females; 57 [34\%] males). Figure 1 shows patient age distribution of the chart review. Majority of the patient $(152 / 68$ [90\%]) had prescription treatment for their dry eye before the initiation of the treatment with lifitegrast. Topical cyclosporine $0.05 \%$ (Restasis ${ }^{\circledR}$ ) and topical corticosteroids were the most prescribed pharmaceutical treatment, and only 36 patients $(21 \%)$ were naïve

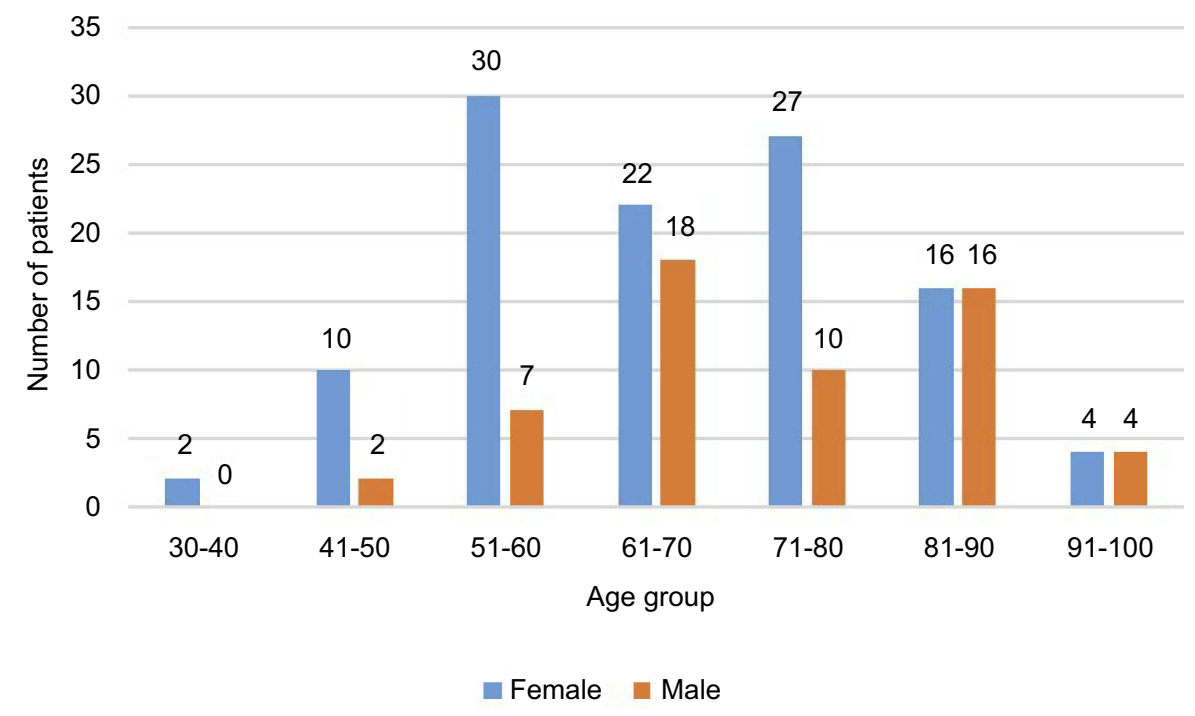

Figure I Patient age distribution of the chart review. 
to any treatment for DED. Prior to lifitegrast treatment, patient-reported DED symptoms included eye dryness (148/168 [88\%]); fluctuation in vision (134/168 [80\%]); tearing (122/168 [73\%]); foreign body sensation (162/168 [97\%]); itching (157/168 [93\%]); eye pain (87/168 [52\%]); grittiness (152/168 [90\%]) (Figure 2). There were 4 subjects who reported contact lens intolerance.

Lifitegrast ophthalmic solution 5\% was prescribed to all 168 patients. Patients were instructed to dose the drop twice a day, once in the morning and once in the evening. At the regular follow-up visits at 1, 2, 3 and 6 months, patients were evaluated through a standard questionnaire and procedures established at the sites. Most of the patients used concurrent medications during the 6-month treatment period with lifitegrast: artificial tears (132/168 [79\%]); lubricant ointment (42/168 [25\%]) and punctal plugs (39/168 [23\%]). There were 5 patients who continued to use topical cyclosporine $0.05 \%$ (Restasis $^{\circledR}$ ) concurrently during the 6-month-treatment period with lifitegrast.

Almost all the patients reported varying degrees of improvement in their dry eye symptoms (166/168) measured with the 4 categories: significant, moderate, minor and no improvement as described in the Patients and methods section. Ninety-four patients $(56 \%)$ rated their improvement as significant overall, 61 patients (36\%) rated as moderate and 11 patients $(6.5 \%)$ as minor. There were only two patients who reported no improvement after 6 months of treatment with lifitegrast (Figure 3).

All patients were asked to recall the onset of their symptom improvement on a monthly grid. Majority of the patients $[121 / 168(72 \%)]$ reported that symptom improvement started at 1 month and 43 patients $(26 \%)$ reported symptom improvement started after 2 months of lifitegrast treatment. Two patients reported that symptom improvement started 3 months after treatment initiation.

The retrospective analysis also found that there were significant improvements in several clinical signs associated with dry eye after 6 months of lifitegrast treatment. SPK was improved in 142 patients from baseline. Conjunctival staining was improved in 27 patients. Conjunctival hyperemia, another important sign often associated with DED, was observed in 161 patients before the start of the treatment and improvement was observed in 138 patients (85\%). Another interesting observation was that among the 50 patients who had tear debris prior lifitegrast treatment significant reduction was seen in 37 patients (74\%). One clinical sign that showed little to no improvement was the tear meniscus level. Only 20 patients (12\%) showed improvement measured with fluorescein strip. (Table 1)

In general, lifitegrast ophthalmic solution 5\% was well tolerated by all 168 patients reviewed. At the post-treatment visits, patients were asked to report 12 different categories of side effects: burning; installation site reaction; blurry vision; bad taste (dysguesia); redness; eye irritation; headache; increased lacrimation; eye discharge; sinusitis; eye pruritus and eye discomfort. The most reported adverse events (>5\%) were dysgeusia (16\%); blurry vision (10\%); burning $(7 \%)$; instillation site reaction $(6.5 \%)$ and transient conjunctival hyperemia (8.3\%). (Table 2) Dysgeusia was the most complained side effect. The blurred vision after lifitegrast drop instillation lasted $<5$ mins for most of the patients who experienced the side effect.

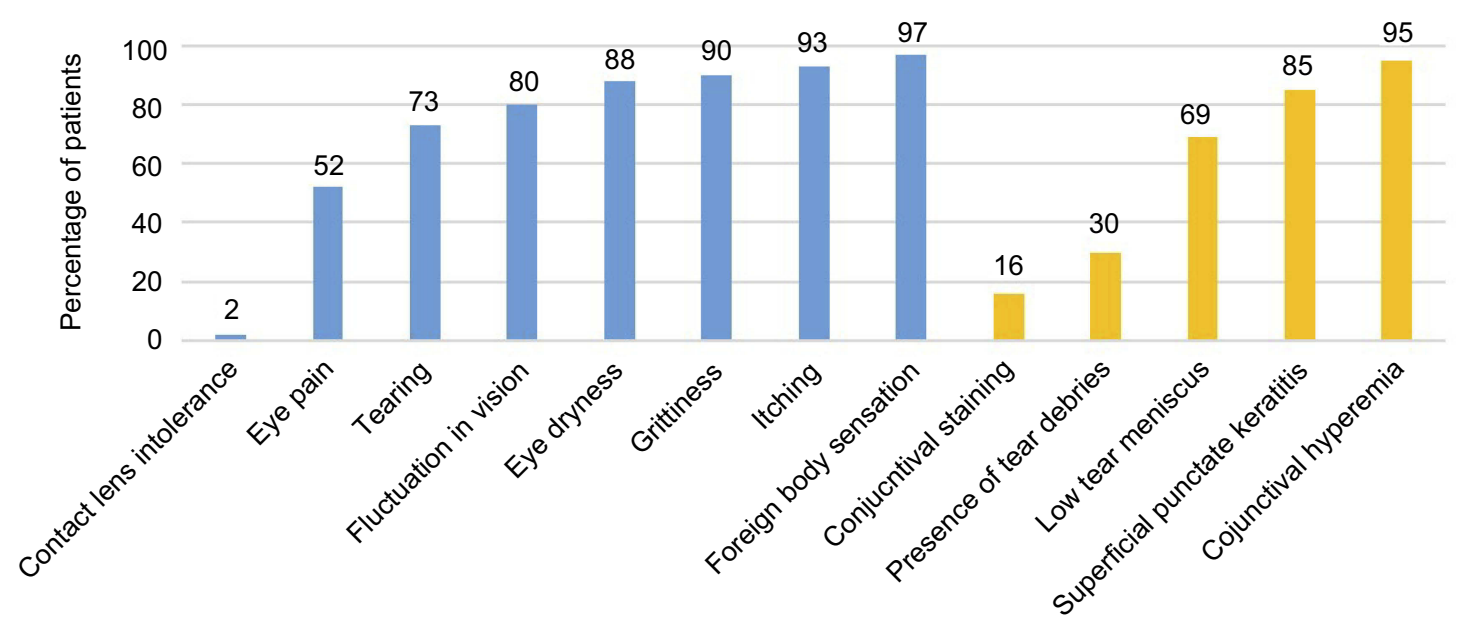

Patient reported symtoms and observed clinical signs at the baseline

Figure 2 Baseline dry eye symptoms (blue bars) and clinical signs (yellow bars). 


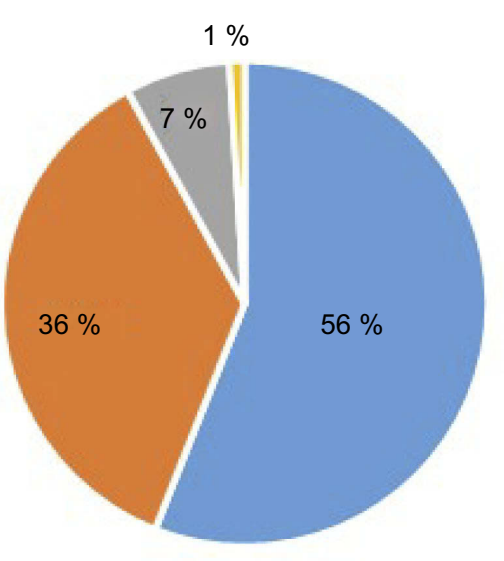

- Significant improvement

- Moderate improvement

- Minor improvement

No

improvement

Figure 3 Improvement in patient-reported symptoms after 6-month treatment with lifitegrast.

Notes: Patients were asked to rate the overall improvement of their eye symptoms at the 6-month visit. Treatment effects were categorized into the 4 categories using a VAS: 0 as no improvement; $1-3$ as minor improvement; $4-6$ as moderate improvement and 7-10 as significant improvement. See Patients and methods section.

Table I Improvement of clinical signs from baseline after 6-month treatment with lifitegrast

\begin{tabular}{|l|l|}
\hline Evaluated clinical signs & $\begin{array}{l}\text { Improvement from baseline } \\
\text { n (improved)/n (baseline) (\%) }\end{array}$ \\
\hline Superficial punctate keratitis & I42/142 (100) \\
Conjunctival staining & $27 / 27(100)$ \\
Conjunctival hyperemia & $138 / 161(85)$ \\
Presence of tear debris & $37 / 50(74)$ \\
Increased tear meniscus & $14 / 116(12)$ \\
\hline
\end{tabular}

Table 2 Summary of most reported (>5\%) adverse events

\begin{tabular}{|l|l|}
\hline $\begin{array}{l}\text { Most frequent adverse } \\
\text { event }\end{array}$ & $\begin{array}{l}\text { Lifitegrast-treated patients, } \\
\mathbf{n}(\%) \\
\mathbf{n = 1 6 8}\end{array}$ \\
\hline Bad taste (dysgeusia) & $28(16)$ \\
Blurry vision & $16(9.5)$ \\
Burning & $13(7)$ \\
Installation site reaction & $11(6.5)$ \\
Eye irritation & $12(7)$ \\
Transient redness & $14(8.3)$ \\
Eye discomfort & $12(7)$ \\
Eye irritation & $12(7)$ \\
\hline
\end{tabular}

Note: Patients were asked to report 12 categories of adverse events, see Patients and methods section.

\section{Discussion and conclusion}

Lifitegrast ophthalmic solution 5\% offers a new treatment option for patients with DED and has generated much interest among eye care professionals since it was approved in
$2016 .{ }^{8,9}$ In this retrospective chart review, we evaluated the treatment outcome and adverse events of 6-month treatment with lifitegrast in patients with moderate-to-severe symptomatic DED. Moderate-to-significant improvement in patientreported symptoms were seen in most of the patients reviewed, and improvements in selected clinical signs (SPK, conjunctival staining and hyperemia) were also observed. The high percentage of symptom improvement seen in this study is consistent with the results of a responder analysis of the OPUS- 2 and OPUS- 3 clinical trials in which approximately $40 \%$ of the subjects reported significant symptom improvement ( $>70 \%$ from baseline) in eye dryness score after 3 months of treatment. ${ }^{6,10,11}$ As a matter of fact in these two large registration trials eye dryness score continued to improve through the 3-month treatment period without sign of plateauing. ${ }^{6,11}$ In this chart review, approximately $56 \%$ of the patients reported significant improvement after 6 months of treatment with lifitegrast, suggesting continued treatment benefits.

Most of the patients in this study were treated with over-the -counter (OTC) and prescription medications for their DED prior to the initiation of the lifitegrast therapy and continued their medications concurrently. There were 5 patients in this chart review who had been treated with cyclosporine ophthalmic emulsion for over a year but remained symptomatic. DED symptoms were significantly improved among these patients who were on lifitegrast and cyclosporine concomitantly, and the side effects reported were similar to the rest of the patients reviewed. Since cyclosporine ophthalmic emulsion is widely used in the treatment of DED the potential clinical benefits with concurrent use of lifitegrast is likely an area of high interests among eye care professionals. Well-designed, prospective trials are needed.

One interesting observation was the improvement of tear debris in some patients reviewed. Excessive tear debris is a common sign of meibomian gland dysfunction (MGD), and the reduction in tear debris might suggest that 6-month treatment with lifitegrast was beneficial for patients with varying degrees of MGD. In the lifitegrast registration clinical trial patients with MGD were not excluded from enrollment and no MGD-related treatment outcome was measured either. A well-designed study is warranted to further evaluate the efficacy of lifitegrast treatment in this highly important MGD population.

One limitation of this study is that the patient population at our practices were primarily elderly. Less than $10 \%$ of the charts reviewed were of patients younger than 50 years old. Younger population is often associated with 
high screen time which is a trigger as well as the cause of DED among this age group. The increasing percentage of male patients with age in this chart review is not of any significant epidemiology meaning and it simply reflects the unique patient population of the practices involved in this study.

In conclusion, this chart review shows that prolonged treatment with lifitegrast ophthalmic solution $5 \%$ beyond 3 months continued to improve both symptoms and signs of DED.

\section{Disclosure}

Dr Eric Donnenfeld has been a consultant for Shire, the manufacturer of Xiidra (lifitegrast ophthalmic solution 5\%). Dr Richard Najac reports personal fees from Shire outside the submitted work. Dr Eric Donnenfeld reports personal fees from Shire during the conduct of the study and personal fees from Allergan and Bausch \& Lomb outside the submitted work. The authors report no other conflicts of interest in this work.

\section{References}

1. Craig JP, Nichols KK, Nichols JJ, et al. TFOS DEWS II Definition and Classification Report. Ocular Surf. 2017;15(3):276-283.
2. Perez VL, Pflugfelder SC, Zhang S, Shojaei A, Haque R. Lifitegrast, a novel integrin antagonist for treatment of dry eye disease. Ocular Surf. 2016;14(2):207-215. doi:10.1016/j.jtos.2016.01.001

3. Pflugfelder SC, Stern ME, Zhang S, Shojaei A. LFA-1/ICAM-1 interaction as a therapeutic target in dry eye disease. $J$ Ocul Pharmacol Thera. 2016;33(1):5-12. doi:10.1089/jop.2016.0105

4. Holland EJ, Whitley WO, Sall K, et al. Lifitegrast clinical efficacy for treatment of signs and symptoms of dry eye disease across three randomized controlled trials. Curr Med Opin. 2016;32 (10):1759-1765. doi:10.1080/03007995.2016.1210107

5. Donnenfeld ED, Karpecki PM, Majmuda PA, et al. Safety of lifitegrast ophthalmic solution $5.0 \%$ in patients with dry eye disease: a 1-Year, multicenter, randomized, placebo-controlled study. Cornea. 2016;35(6):741-748. doi:10.1097/ICO.0000000000000803

6. Holland EJ, Luchs J, Karpecki PM, et al. Lifitegrast for the treatment of dry eye disease: results of a phase III randomized, double-masked, placebo-controlled trial (OPUS-3). Ophthalmology. 2017;124 (1):53-60. doi:10.1016/j.ophtha.2016.09.025

7. Lienert JP, Tarko L, Uchino M, Christen WG, Schaumberg DA. Long-term natural history of dry eye disease from the patient's perspective. Ophthalmology. 2016;123(2):425-433. doi:10.1016/j. ophtha.2015.10.011

8. Jones L, Downie L, Korb D, et al. TFOS DEWS II management and therapy report. Ocular Surf. 2017;15(3):575-628. doi:10.1016/j. jtos.2017.05.006

9. Xiidra Prescribing Information. Shire, July 2016.

10. Tauber J, Holland EJ, Baudouin C et al. Response to lifitegrast $5.0 \%$ for the treatment of dry eye disease: responder analysis for reductions in eye dryness score in two phase 3 randomized controlled trials. Presented at ISOPT Clinical March 1-3, 2018; Tel Aviv, Israel.

11. Tauber J, Karpecki P, Latkany R, et al. Lifitegrast ophthalmic solution $5.0 \%$ versus placebo for treatment of dry eye disease: results of the randomized phase III OPUS-2 study. Ophthalmology. 2015;122 (12):2423-2431. doi:10.1016/j.ophtha.2015.08.001
Clinical Ophthalmology

\section{Publish your work in this journal}

Clinical Ophthalmology is an international, peer-reviewed journal covering all subspecialties within ophthalmology. Key topics include: Optometry; Visual science; Pharmacology and drug therapy in eye diseases; Basic Sciences; Primary and Secondary eye care; Patient Safety and Quality of Care Improvements. This journal is indexed on PubMed
Central and CAS, and is the official journal of The Society of Clinical Ophthalmology (SCO). The manuscript management system is completely online and includes a very quick and fair peer-review system, which is all easy to use. Visit http://www.dovepress.com/ testimonials.php to read real quotes from published authors. 